\title{
Effect of fat mass index, fat free mass index and body mass index on childhood blood pressure: a cross-sectional study in south China
}

\author{
Huijing He ${ }^{1}$, Li Pan ${ }^{1}$, Jianwei Du², Yuming Jin ${ }^{2}$, Li Wang ${ }^{1}$, Pengben Jia ${ }^{2}$, Guangliang Shan ${ }^{1}$ \\ ${ }^{1}$ Department of Epidemiology and Statistics, Institute of Basic Medical Sciences, Chinese Academy of Medical Sciences, School of Basic Medicine, \\ Peking Union Medical College, Beijing, China; ${ }^{2}$ Hainan Provincial Center for Disease Control and Prevention, Haikou, China \\ Contributions: (I) Conception and design: G Shan, H He; (II) Administrative support: G Shan, L Pan; (III) Provision of study materials or patients: \\ G Shan, J Du; (IV) Collection and assembly of data: G Shan, L Pan, J Du, Y Jin, L Wang, P Jia; (V) Data analysis and interpretation: H He; (VI) \\ Manuscript writing: All authors; (VII) Final approval of manuscript: All authors. \\ Correspondence to: Prof. Guangliang Shan. 5 Dongdan-Santiao, Dongcheng District, Beijing 100005, China. Email: guangliang_shan@163.com.
}

Background: Evidence on how different body composition indexes, such as body mass index (BMI), fat mass index (FMI) and fat free mass index (FFMI), influence blood pressure (BP) in Chinese children and adolescents is sparse. The aim of this study is to assess the effect of different body composition on BP in participants aged 7-18 in south China.

Methods: A cross-sectional study was conducted in 2013. A total of 1,609 children and adolescents from urban and rural areas in Hainan Province, South China, were selected using multi-stage stratified cluster sampling method. Each participant underwent anthropometric and BP tests. BMI, FMI and FFMI were calculated and analyzed as body composition indexes.

Results: In the population level stratified by sex, FFMI had the highest r-value (Partial Spearman correlations) of correlation with systolic $\mathrm{BP}$ in boys $\left(\mathrm{r}_{\mathrm{s}}=0.305, \mathrm{P}<0.001\right)$ and girls $\left(\mathrm{r}_{\mathrm{s}}=0.237, \mathrm{P}<0.001\right)$. FMI was found to have the highest $r$-value of correlation with DBP in boys $\left(r_{s}=0.154, P<0.001\right)$. The quantile regression model indicated that, within each age group, all body composition indexes was positively associated with systolic BP, and FFMI has the greatest value of regression coefficient (4.38, 95\% CI: 3.81-4.95). But for diastolic BP, positive associations with BMI, FMI and FFMI were only observed in the 11-12 years group. Restrict cubic spline models showed that there were both linear and non-linear relations between BMI, FMI and BP, but for FFMI, there was only linear association with BP.

Conclusions: The relation between BP and FFMI was stronger than with BMI and FMI. Combined measurement of body composition should be considered to help health professionals interpret body mass data from children and adolescents with varied stature and age.

Keywords: Children; adolescent; body composition; fat mass; blood pressure (BP)

Submitted Oct 08, 2020. Accepted for publication Feb 05, 2021.

doi: $10.21037 /$ tp-20-325

View this article at: http://dx.doi.org/10.21037/tp-20-325 


\section{Introduction}

Previous evidence emphasized that adult cardiovascular disease begins in child $(1,2)$. With high hypertension burden, China has an increasing prevalence of childhood elevated blood pressure (BP) along with the increasing prevalence of overweight and obesity $(3,4)$.

Excess adiposity was considered as a most important risk factor for elevated BP (5). By virtue of its convenience of measurement, body mass index (BMI) is the most common method used to measure overweight and obesity. However, BMI does not allow the measure of body composition, as it does not differentiate between fat-free mass and fat mass (6), which are important factors of malnutrition impacting disease outcome (7). In childhood, $\mathrm{BMI}$ is a poor indicator of body composition (8). Using BMI alone may underestimate the risk of some nutrition-related disease (9). Body composition is the relative proportion of muscle, fat, bone, water, and other components of the body (10). Fat mass index (FMI) and fat free mass index (FFMI) first put forward by VanItallie et al. in 1990 (11), can identify whether the excess body weight is due to fat mass (FM) or fat free mass (FFM) or both. As stature-adjusted index, FMI and FFMI enable the comparisons among people with different body size. The combined measurement of BMI, FMI and FFMI could promote the understanding of which body composition index is the strongest predictor of BP.

Age-related increase in BP is now recognized as a universal feature of human aging (12). Similarly, in children and adolescents, previous study also observed an agerelated trend in BP $(13,14)$. Limited by the sample size, few studies could conduct age-stratified analyses to assess the heterogeneity of the effect of body composition on BP. The present knowledge on the age-related association between body composition and BP are mostly in adults $(15,16)$. In addition, as there was sexual dimorphism in body fat proportion and distribution, understanding whether there is heterogeneity across age and sex in the effect of body composition on BP among children and adolescents is important for clinical practice.

On the consideration of the above, the present study aimed to investigate how body composition affect BP and whether the effect varied across sex and age groups in children and adolescents aged 7-18 in Hainan Province, south China. The current analyses use BMI, FMI and FFMI as the measurement of body composition because they are all standardized by stature, the contribution of both fat mass and fat-free mass to BMI can be easily assessed (17). We also explored the potential modification effect of age on the association between different body composition indexes on BP by age-stratified analyses.

We present the following article in accordance with the STROBE reporting checklist (available at http://dx.doi. org/10.21037/tp-20-325).

\section{Methods}

\section{Study design and population}

This cross-sectional study is based on the data derived from several sites in Hainan Province, south China, and using same sampling method with our previous studies $(18,19)$. Briefly, from Nov to Dec 2013, a multi-stage stratified clustering sampling method was used to select subjects. Firstly, the provincial capital city, one midsize city and two counties were selected from Hainan province based on their economic status measured by local gross domestic product (GDP). Secondly, districts were selected from cities, and rural townships were selected from counties. In the final stage, communities were selected from districts in urban areas, whereas villages were selected from townships in rural areas. Individuals aged from 7 to 18 who had lived in current residence for at least one year were eligible to participate. Subjects who were diagnosed with hypertension or took medication of hypertension were excluded.

The study was conducted in accordance with the Declaration of Helsinki (as revised in 2013). The study was approved by the Bioethical Committee of Institute of Basic Medical Sciences, Chinese Academy of Medical Sciences (NO. 029-2013) and informed consent was taken from all the participants (or their parents) before the survey.

As in this study, BP value was the main interest and set as dependent variable, the sample size was calculated based the Eq. [1]:

$$
n=Z_{\alpha}^{2} S^{2} / d^{2}
$$

Alpha $(\alpha)$ was the significance level, which was set as $0.05, \mathrm{~S}$ was the standard deviation of childhood SBP which was estimated as $12 \mathrm{mmHg}$ by our previous study (18), and $\mathrm{d}$ was tolerance error which was estimated as 5 mmHg. The estimated minimum sample size was 22 . We added an additional $20 \%$ to the minimum sample size, factoring in possible noncompliance, and targeted 
28 subjects in each stratum by sex and age group, which was totally $28 \times 2 \times 6=336$. Finally, a total of 1,609 children and adolescents living in Hainan province met the recruitment criteria and were enrolled in the study.

\section{Data collection and measurements}

A structured face-to-face questionnaire interview was conducted by trained staff. Demographic information, such as sex, age, and residential areas, was obtained through the parents of the subjects.

Before the survey, all participants were required to maintain their regular lifestyle for at least one week and fast overnight for at least $8 \mathrm{~h}$ (10). Physical examinations were conducted and information on anthropometry and BP were collected. Stature was measured to nearest $0.1 \mathrm{~cm}$ using a fixed stadiometer. Weight, body fat percentage (\%BF), fat mass (FM, kg) and fat free mass (FFM, kg) were measured by body composition analyzer (TANITA BC-420, Tokyo, Japan), with the accuracy to decimal level. The device was calibrated daily with a standard according to manufacturer's instruction (20).During the anthropometric measurements, participants were light clothing and barefoot. BMI was calculated as weight in kilograms divided by the square of stature in meters $\left(\mathrm{kg} / \mathrm{m}^{2}\right)$. FMI was calculated as FM in kilograms divided by stature in square meters $\left(\mathrm{kg} / \mathrm{m}^{2}\right)$ and FFMI was fat free mass in kilograms divided by stature in square meters $\left(\mathrm{kg} / \mathrm{m}^{2}\right)$.

Systolic blood pressure (SBP) and diastolic blood pressure (DBP) were measured on the right arm using a digital BP measuring device (Omron HEM-907, Tokyo, Japan) with children customized cuff size after one subject had rested for at least five minutes in a sitting position. Three readings were measured with one-minute interval and the average value was recorded.

\section{Statistical analyses}

Data with missing values on body composition measurements or BP were excluded from the final analyses. The final sample contained 1,608 subjects.

Summary results were presented as median (Interquartile range, IQR) for continuous data and counts (percentage, $\%$ ) for categorical data. For continuous variables, Wilcoxon sum-rank test (because of data non-normal distribution) was used to perform two-group comparisons. CochranArmitage trend test was used to explore the potential age trend of body composition and BP within each sex group. Partial Spearman correlation coefficients were used to assess the correlation between BMI, FMI, FFMI and BP in all study participants. Quantile regression models (in which median values were compared) were used to investigate the age-specific association between body composition and BP (both SBP and DBP). The regression coefficient represents the effect size of the association between exposure and outcome, the larger the value is, the stronger the association would be.

In addition, we examined the possible non-linear relationship between body composition and BP using restricted cubic splines (RCS). Tests for non-linearity used the likelihood ratio test, and compared the model with only the linear term to the model with the linear and the cubic spline terms. A two-tailed $\mathrm{P}$ value $<0.05$ indicated statistical significance. All statistical analyses were performed using SAS 9.4 (SAS Institute Inc. Cary, NC, USA).

\section{Results}

\section{General characteristics of the participants}

The general characteristics of the 1,608 children and adolescents stratified by sex were shown in Table 1 . The median of age was $12.86(5.31)$ years and $57.6 \%(927 / 1,608)$ were girls. All the anthropometric measurements differed by sex except for residential areas and BMI. Stature, weight, FM, \%BF, FMI, FFM, FFMI and SBP were higher in boys, whereas DBP was higher in girls.

\section{Age trends of body composition indexes and BP}

In both sexes, the mean levels of all body composition indexes and BP maintained increasing trends ( $\mathrm{P}$ for trend $<0.001)$ with age. Boys and girls had similar BMI level through all age groups, but higher FMI and lower FFMI in girls than in boys. The sex difference of SBP was between the peak age range of 17-18 (119.97/109.09) and the trough at age of 7-8 (95.61/96.36). But for DBP, there was no sex difference in most age groups (Table 2). The RCS regression models revealed that, for SBP and DBP, there were also non-linear trends with age increase (non-linear $\mathrm{P}$ value for $\mathrm{SBP}=0.0002$, for $\mathrm{DBP}<0.001$ ).

\section{The relationship between BP and body composition indexes}

BMI, FMI and FFMI were all found positively correlated 
Table 1 Characteristics of the children and adolescents in Hainan Province, China.

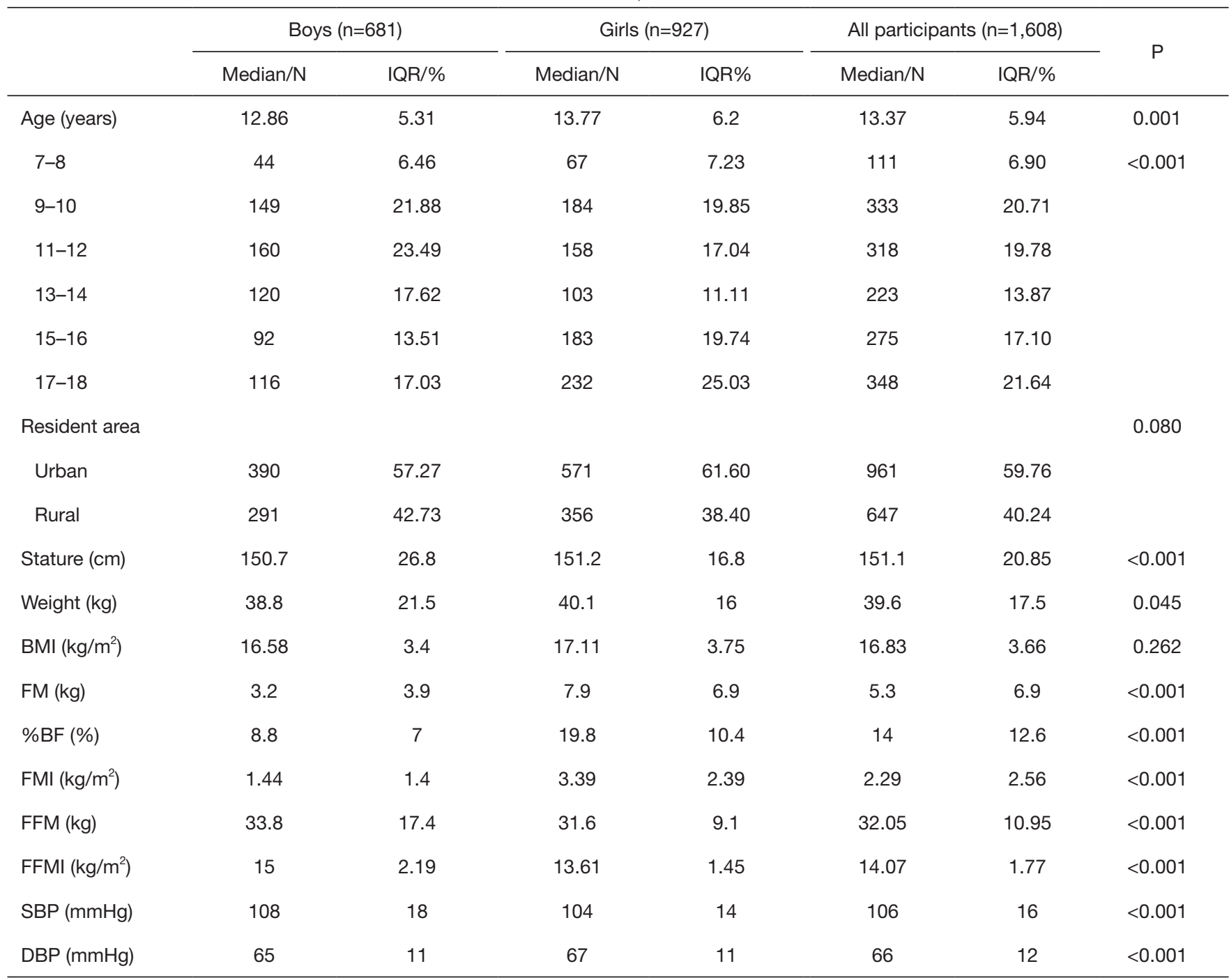

BMI, body mass index; FM, fat mass; \%BF, body fat percentage; FMI, fat mass index, calculated as FM in kilograms divided by stature in square meters $\left(\mathrm{kg} / \mathrm{m}^{2}\right)$; FFMI, fat free mass index, calculated as fat free mass in kilograms divided by stature in square meters $\left(\mathrm{kg} / \mathrm{m}^{2}\right)$. SBP, systolic blood pressure; DBP, diastolic blood pressure. IQR, Interquartile range.

with SBP in both sexes. BMI and FMI were also found positively correlated with DBP in boys and girls, but no correlation was found between FFMI and DBP in either sex. Among the three indexes, FFMI had the highest Spearman correlation coefficient ( $r$-value) in the relation with SBP in both boys $\left(r_{s}=0.305, P<0.001\right)$ and girls $\left(r_{s}=0.237, P<0.001\right)$. The age- and residential-adjusted correlation coefficients of BMI, FMI and FFMI with DBP were weaker than those of SBP. FMI was found to have the highest r-value of correlation with DBP in boys $\left(\mathrm{r}_{\mathrm{s}}=0.154, \mathrm{P}<0.001\right)$, but lowest in girls $\left(\mathrm{r}_{\mathrm{s}}=0.010\right.$, $\mathrm{P}=0.002$ ) (Table 3).

All composition indexes were found associated with SBP and DBP, after the adjustment of sex, age and residential areas (all $\mathrm{P}<0.001$, Figure 1). To further explore factors which may influence the age-related trend of BP increases, the association of BP and body composition indexes were analyzed in each age group. BMI, FMI and FFMI were associated with SBP in age groups above 8 . The associations of body composition indexes with DBP seemed weaker 
Table 2 Age-specific body fat and blood pressure in children and adolescents aged 7-18 in Hainan Province, China

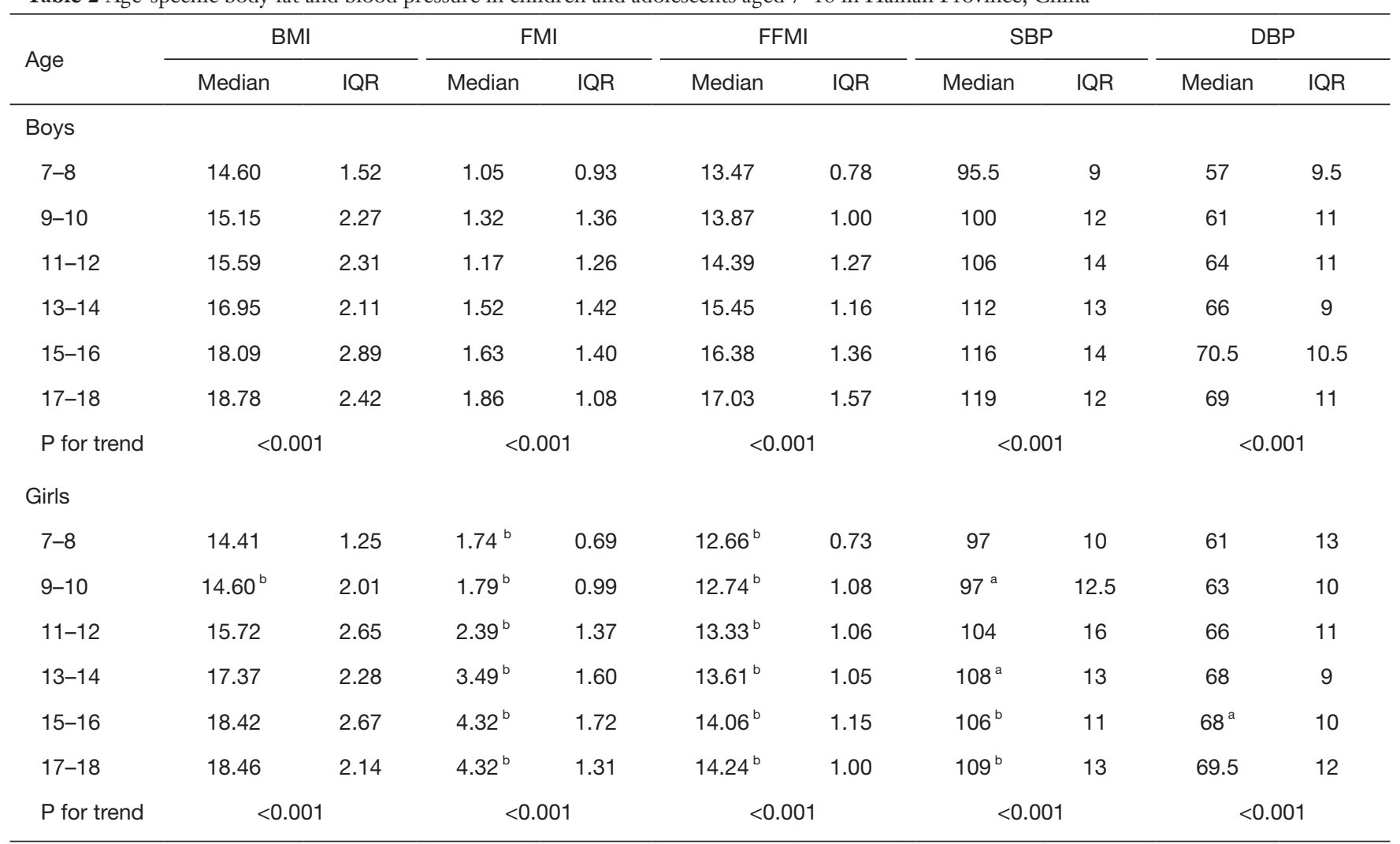

${ }^{a}$, $\mathrm{P}$ value less than 0.05 for sex difference within age group; ${ }^{\mathrm{b}}, \mathrm{P}$ value less than 0.001 for sex difference within age group; BMI, body mass index; FM, fat mass; \%BF, body fat percentage; FMI, fat mass index, calculated as FM in kilograms divided by stature in square meters $\left(\mathrm{kg} / \mathrm{m}^{2}\right)$; FFMI, fat free mass index, calculated as fat free mass in kilograms divided by stature in square meters $\left(\mathrm{kg} / \mathrm{m}^{2}\right)$. SBP, systolic blood pressure; DBP, diastolic blood pressure. IQR, Interquartile range.

Table 3 The partial correlation between body fat indexes and blood pressure

\begin{tabular}{|c|c|c|c|c|c|c|}
\hline & \multicolumn{3}{|c|}{ Boys } & \multicolumn{3}{|c|}{ Girls } \\
\hline \multicolumn{7}{|c|}{ SBP } \\
\hline$r_{s}$ & 0.304 & 0.262 & 0.305 & 0.207 & 0.161 & 0.237 \\
\hline $\mathrm{P}$ & $<0.001$ & $<0.001$ & $<0.001$ & $<0.001$ & $<0.001$ & $<0.001$ \\
\hline \multicolumn{7}{|c|}{ DBP } \\
\hline$r_{s}$ & 0.128 & 0.154 & 0.066 & 0.086 & 0.010 & 0.047 \\
\hline $\mathrm{P}$ & $<0.001$ & $<0.001$ & 0.085 & 0.009 & 0.002 & 0.155 \\
\hline
\end{tabular}

Adjusted for age and residential areas. $\mathrm{r}_{\mathrm{s}}$, coefficient of Spearman correlation. BMl, body mass index, $\mathrm{kg} / \mathrm{m}^{2} ; \mathrm{FMl}$, fat mass index, $\mathrm{kg} / \mathrm{m}^{2}$; FFMI, fat free mass index, $\mathrm{kg} / \mathrm{m}^{2}$; SBP, systolic blood pressure, $\mathrm{mmHg}$; DBP, diastolic blood pressure, $\mathrm{mmHg}$. 

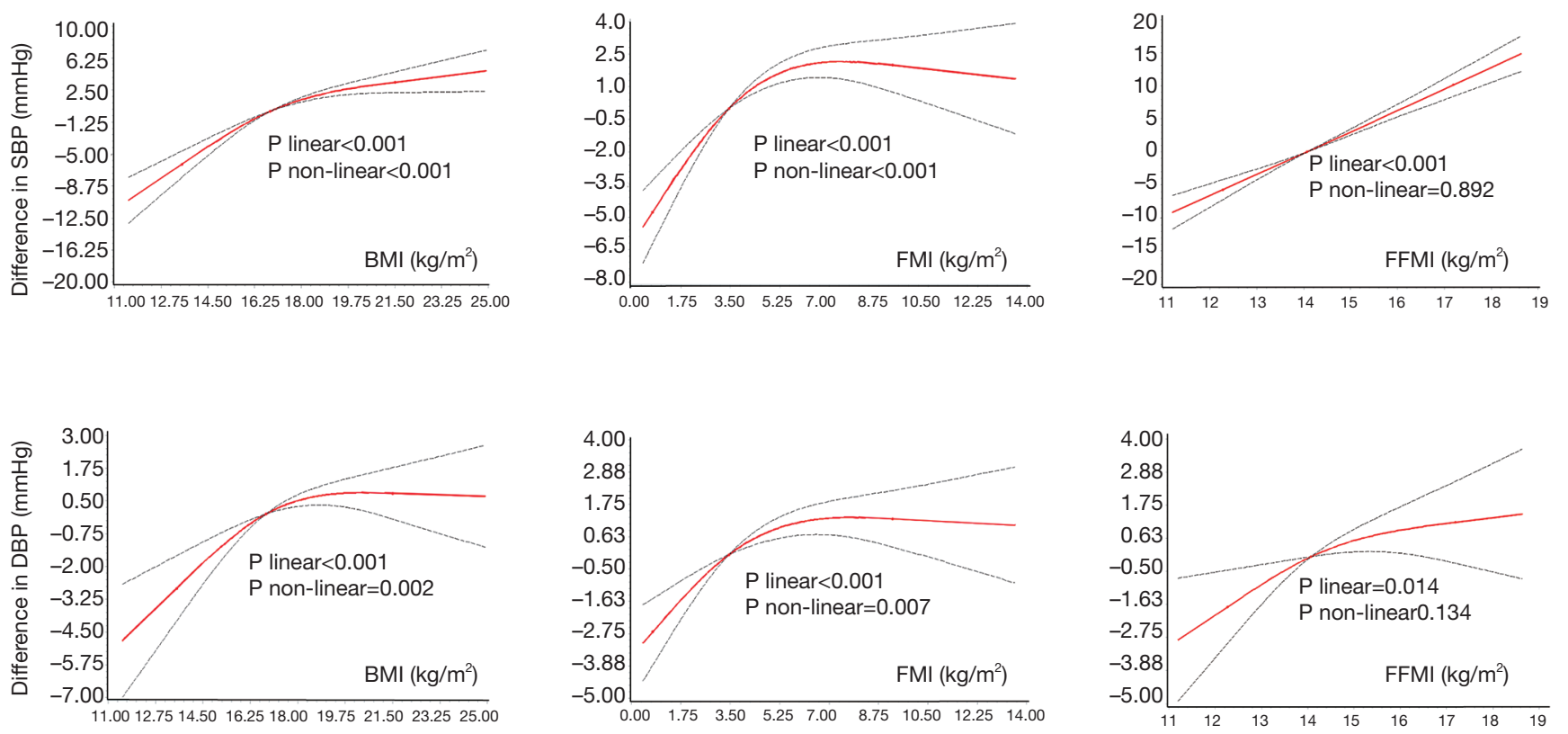

Figure 1 The restricted cubic spline for the association between body composition indexes (measured by BMI, FMI and FFMI) and difference in blood pressure (SBP and DBP). The lines represent adjusted difference based on restricted cubic splines for the level of body composition in multi-variable linear and non-linear regression models. Adjusted factors were age, sex and residential areas. BMI, body mass index, $\mathrm{kg} / \mathrm{m}^{2}$; FMI, fat mass index, $\mathrm{kg} / \mathrm{m}^{2}$; FFMI, fat free mass index, $\mathrm{kg} / \mathrm{m}^{2}$; SBP, systolic blood pressure, mmHg; DBP, diastolic blood pressure, $\mathrm{mmHg}$. The solid lines represent the predicted value, and the dash lines were their $95 \%$ confidence interval.

comparing with those with SBP. Although the overall tests of the association between BMI, FMI, FFMI and DBP all showed positive results, the age-specific associations presented that only in the 11-12 age group, all the three body composition indexes were associated with DBP. There was also positive association between FMI and DBP in the 17-18 age group (Table 4).

\section{Discussion}

This cross-sectional study was based on a communitybased population involving 1,608 Chinese children and adolescents and demonstrated that magnitude of changes in body composition measured by bioelectrical impedance analysis (BIA) varied by sex and age. To the best of our knowledge, this is the first study investigating age-related association between BMI, FMI and FFMI and BP in Chinese children and adolescents.

There are methods available for measuring body composition. Techniques with high accuracy for estimating body fat such as dual-energy X-ray absorptiometry (DXA) and magnetic resonance imaging (MRI) are operationally costly and require expense training (21). Thus, alternative methods such as BIA with low operational costs is necessary in health practice and has been widely used to evaluate body composition in both clinical and epidemiological studies (22). BIA has high reproducibility (23), and there was a strong relationship between $\% \mathrm{BF}$ derived from the BIA system and that derived from DXA (10). Thus, the magnitude of body composition indexes measured by BIA in the current study was more likely to reflect the true level of body mass.

Previous studies have revealed sex difference in the growth of body composition with aging $(24,25)$, but evidence derived from children and adolescents in China is sparse. The current study suggested that sex difference existed both in body composition indexes and BP levels. Girls had higher FMI but lower FFMI than boys in almost each age group, but BMI showed no difference between sexes. Dallaire (26) stated that there was sex difference on cardiovascular function from early fetal life to late adolescence, but the multifactorial influence of sex has not been fully understood. The current study revealed that there was sex difference on BP in older age 
Table 4 Adjusted median regression coefficients of body composition indexes by SBP in children and adolescents

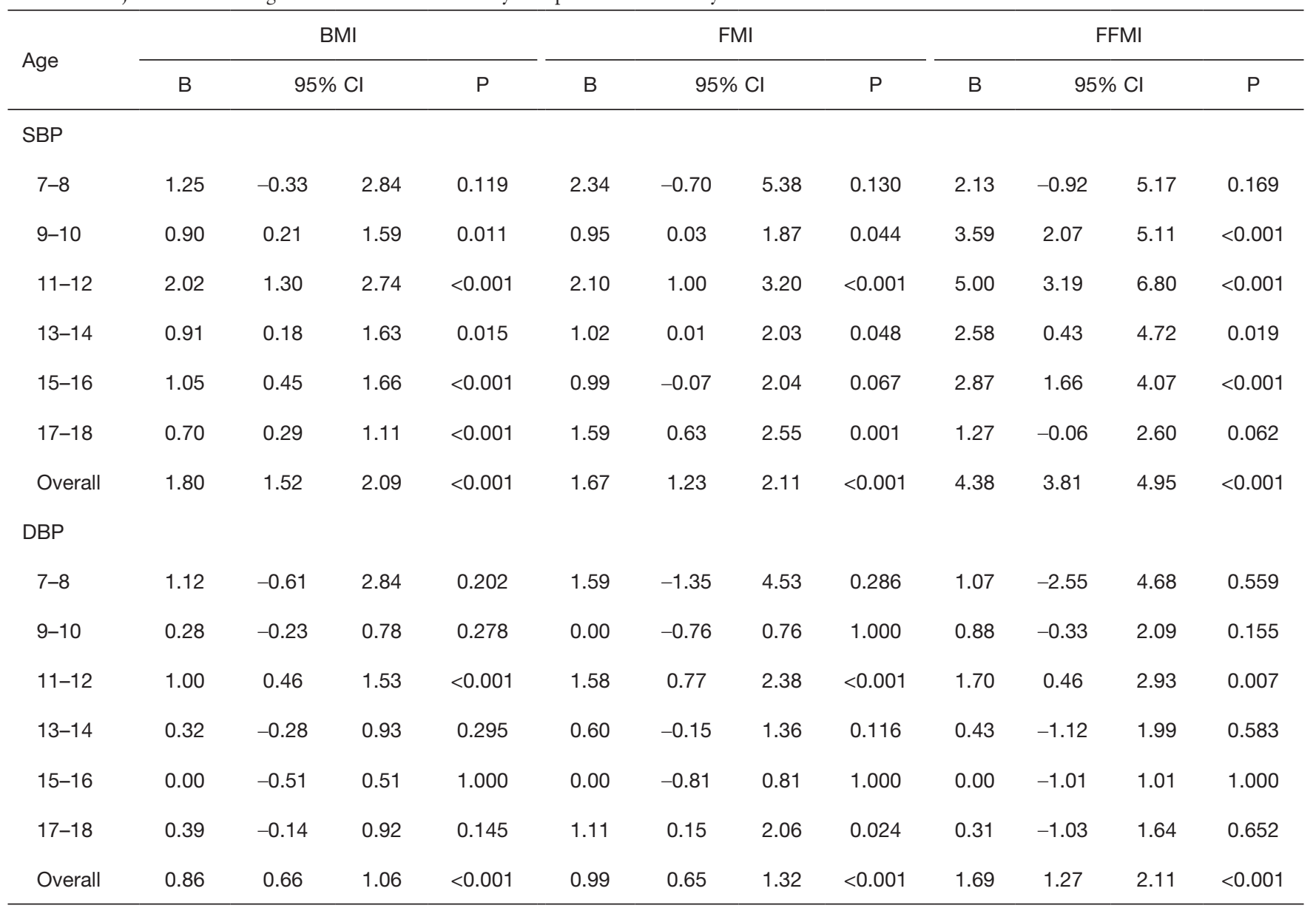

$\mathrm{B}$ was the regression coefficient of quantile regression models and was adjusted for sex and resident areas. BMl, body mass index, $\mathrm{kg} / \mathrm{m}^{2}$; FMI, fat mass index, $\mathrm{kg} / \mathrm{m}^{2}$; FFMI, fat free mass index, $\mathrm{kg} / \mathrm{m}^{2}$; SBP, systolic blood pressure, $\mathrm{mmHg}$; DBP, diastolic blood pressure, $\mathrm{mmHg}$.

group in SBP but not on DBP. This may be attribute to the increase of androgens such as testosterone, which was believed to have effect on elevating BP, in boys during their puberty (27).

The association between $\mathrm{BMI}$ and $\mathrm{BP}$ has been well documented in children and adolescents by some epidemiological studies $(28,29)$. For example, for Chinese youths, Yang's study (30) showed that BMI was found positively associated with high $\mathrm{BP}$ in both sexes in children and adolescents aged 7-17. Zhang's study (31) also demonstrated that higher BMI quartiles was associated with relatively higher $\mathrm{BP}$, comparing with the first quartile. However, in other ethnicities, such as the Bogalusa Heart Study (32), in which children and adolescents aged 5-17 were recruited in Louisiana, showed that neither DBP nor
SBP increased with the increases of BMI, in other words, BP levels didn't parallel changes in obesity measured by BMI. As measured in our study, BMI was associated with BP levels, and the RCS models illustrated that for both SBP and DBP, there were linear and non-linear relations with BMI (all $\mathrm{P}$ value $<0.01$ ). However, after stratified by age, the associations between BMI and DBP lost their statistical significance except for age 11-12. Thus, there may be agerelated association between BMI and DBP.

As BMI can be considered as the sum of FMI and FFMI, the change on BP due to BMI can be further explained by the contribution of FMI or FFMI. Nevertheless, few studies have explored the association between FMI or FFMI and BP in children and adolescents. The advantage of investigating both the effect of FMI and FFMI on BP 
increasing across sex and age groups is that, it could help researchers understand whether the increase or decrease of $\mathrm{BP}$ in particular age group is selectively due to a change in fat mass or fat free mass, or both. The present study demonstrated that all measures of body composition indexes were correlated to $\mathrm{BP}$ at the whole population level, and among them, the relationships between FFMI with BP appealed to be stronger than FMI, indicating that fat free mass may play predominant role in influencing BP.

LA de Hoog et al. (33) explored that increasing body size (measure by FMI and BMI) was observed associated with increased BP in multi-ethnic children aged 5-6. In Zhang's study $(14,34)$, positive relationships between FMI, FFMI and BP were observed among children aged 7-12 years in China. The present study investigated the effect of FMI and FFMI on BP in a wider age range of children and adolescents in China, and furthermore, by age-stratified analysis, we were able to understand the potential age-related association between body composition index and BP. As demonstrated by the quantile regression models, although in the overall population level, all the three body composition indexes were positively associated with DBP, when stratified by age, there were positive association between the three body composition indexes and DBP only in age group 11-12. For SBP, the effect of body composition indexes on $\mathrm{BP}$ was positively observed expect for the 7-8 group. Although body composition indexes were not significantly associated with BP in age 7-8, the insignificance association may be caused by the limited sample size. The RCS models revealed that for both SBP and DBP, there were not only linear, but also non-linear relationships between age and BP. We may expect that the change of BP across age stage was different. But for body composition indexes, such as BMI, FMI and FFMI, there were linear elevated trends as age increase. By this indirect interpretation, combined with age- and sex-stratum analysis using quantile regression models, we may conclude that the effect of body composition on BP is likely to be influenced by age.

As to which body composition index is the strongest predictor of BP, the evidence is limited. Zhang's study $(14,34)$ concluded that the correlation between BP and FMI was stronger than with FFMI and BMI. However, in Zhang's study, \%BF was calculated based on a regression equation (35) but not measured directly and BP was measured differently with ours. The present study suggested that, FFMI possibly had the strongest correlation with
$\mathrm{BP}$ due to its largest magnitude of both partial correlation coefficients and regression coefficients in the regression models.

In addition, the RCS models also suggested that for BMI and FMI, there were both linear and non-linear relations with BP, but for FFMI, there were only linear relations with SBP and DBP. Increased FFMI was associated with elevated BP. Thus, although BMI is useful in evaluating body mass of youths with different stature, other BMI, such as FMI and FFMI, have potential value in helping healthcare professionals interpret body composition data from population with varied stature and age.

There were limitations of the current study. Firstly, our data were cross-sectional, therefore no conclusions regarding causality can be drawn. Secondly, the present study used single day measurements of BP, which could not be able to reflect the true BP level due to the inherent variability or accommodation effect in the individual level (36). However, it is difficult for epidemiological studies to conduct repeated BP measurements. We have requested subjects to rest at least for five minutes in a sitting position to get more precise results of BP. As the purpose of this study was not to estimate the prevalence nor diagnose of high BP, but the general idea of the potential age-related association between body composition and BP level, the conclusions of this study may not be biased. Thirdly, as we did not collect information on other factors that may influence childhood BP such as genetic background, parental socioeconomic status, nutrition factors or physical activities, etc., we are not able to analyze their potential modification or confounding effects.

However, the strength of this study should also be acknowledged, as its conclusion was derived from a representative sample using a multi-stage stratified sampling method, and the relatively large sample size which enabled the age-stratified analysis. More sensitive indexes, rather than the well-studied BMI, correlated with BP, such as FMI and FFMI were explored thoroughly with age-stratified analysis in the present study. Furthermore, we use RCS models to investigate the potential non-linear relations between body compositions and BP for the first time in Chinese children and adolescents.

\section{Conclusions}

Using community-based population data, this study reported that $\mathrm{BP}$ showed an age-related increasing trend, 
and the relation between BP and FFMI was stronger than with BMI and FMI. FMI and FFMI should be considered as useful measurements in helping clinical or healthcare professionals interpret body mass data from children and adolescents with varied stature and age.

\section{Acknowledgments}

We would like to thank all the participants and staff members from Hainan Provincial Centers for Disease Control and Prevention. We also gratefully appreciate Guangjin Zhu, Jing Nai, Ke Wang, Fen Dong and Haiying Gong for their efforts in the field work.

Funding: This study was supported by Beijing Natural Science Foundation (7204279), CAMS Innovation Fund for Medical Sciences (CIFMS), Grant No. 2018-I2M-1-001, 2019-I2M-2-007.

\section{Footnote}

Reporting Checklist: The authors have completed the STROBE reporting checklist. Available at http://dx.doi. org/10.21037/tp-20-325

Data Sharing Statement: Available at http://dx.doi. org/10.21037/tp-20-325

Peer Review File: Available at http://dx.doi.org/10.21037/tp20-325

Conflicts of Interest: All authors have completed the ICMJE uniform disclosure form (available at http://dx.doi. org/10.21037/tp-20-325). The authors have no conflicts of interest to declare.

Ethical Statement: The authors are accountable for all aspects of the work in ensuring that questions related to the accuracy or integrity of any part of the work are appropriately investigated and resolved. The study was conducted in accordance with the Declaration of Helsinki (as revised in 2013). The study was approved by the Bioethical Committee of Institute of Basic Medical Sciences, Chinese Academy of Medical Sciences (NO. 029-2013) and informed consent was taken from all the participants (or their parents) before the survey.

Open Access Statement: This is an Open Access article distributed in accordance with the Creative Commons Attribution-NonCommercial-NoDerivs 4.0 International License (CC BY-NC-ND 4.0), which permits the noncommercial replication and distribution of the article with the strict proviso that no changes or edits are made and the original work is properly cited (including links to both the formal publication through the relevant DOI and the license). See: https://creativecommons.org/ licenses/by-nc-nd/4.0/.

\section{References}

1. Kavey RE, Daniels SR, Lauer RM, et al. American Heart Association Guidelines for Primary Prevention of Atherosclerotic Cardiovascular Disease Beginning in Childhood. Circulation 2003;107:1562-66.

2. Falkner B. The Childhood Role in Development of Primary Hypertension. Am J Hypertens 2018;31:762-9.

3. Ingelfinger JR. The child or adolescent with elevated blood pressure. N Engl J Med 2014;371:1075.

4. Lo JC, Sinaiko A, Chandra M, et al. Prehypertension and hypertension in community-based pediatric practice. Pediatrics 2013;131:e415-24.

5. Anyaegbu EI, Dharnidharka VR. Hypertension in the Teenager. Pediatr Clin North Am 2014;61:131-51.

6. Martinez KE, Tucker LA, Bailey BW, et al. Expanded Normal Weight Obesity and Insulin Resistance in US Adults of the National Health and Nutrition Examination Survey. J Diabetes Res 2017;2017:9502643.

7. Nuttall FQ. Body Mass Index: Obesity, BMI, and Health: A Critical Review. Nutrition Today 2015;50:117-28.

8. Calcaterra V, Pelizzo G, Cena H. BMI Is a Poor Predictor of Nutritional Status in Disabled Children. What Is the Most Recommended Method for Body Composition Assessment in This Pediatric Population? Front Pediatr 2019;7:226.

9. Whitney DG, Miller F, Pohlig RT, et al. BMI does not capture the high fat mass index and low fat-free mass index in children with cerebral palsy and proposed statistical models that improve this accuracy. Int J Obes (Lond) 2019;43:82-90.

10. Jin M, Du H, Zhang Y, et al. Characteristics and reference values of fat mass index and fat free mass index by bioelectrical impedance analysis in an adult population. Clin Nutr 2019;38:2325-32. 
11. VanItallie TB, Yang MU, Heymsfield SB, et al. Heightnormalized indices of the body's fat-free mass and fat mass: potentially useful indicators of nutritional status. Am J Clin Nutr 1990;52:953-9.

12. O'Rourke MF, Nichols WW. Aortic diameter, aortic stiffness, and wave reflection increase with age and isolated systolic hypertension. Hypertension 2005;45:652-8.

13. Zhai Y, Li W, Shen C, et al. Prevalence and Correlates of Elevated Blood Pressure in Chinese Children Aged 6-13 Years: a Nationwide School-Based Survey. Biomed Environ Sci 2015;28:401-9.

14. Zhang YX, Wang SR. Relation of body mass index, fat mass index and fat-free mass index to blood pressure in children aged 7-12 in Shandong, China. Ann Hum Biol 2011;38:313-6.

15. Hübers M, Geisler C, Plachta-Danielzik S, et al. Association between individual fat depots and cardiometabolic traits in normal- and overweight children, adolescents and adults. Nutr Diabetes 2017;7:e267.

16. Li W, He Y, Xia L, et al. Association of Age-Related Trends in Blood Pressure and Body Composition Indices in Healthy Adults. Front Physiol 2018;9:1574.

17. Freedman DS, Wang J, Maynard LM, et al. Relation of BMI to fat and fat-free mass among children and adolescents. Int J Obes (Lond) 2005;29:1-8.

18. He H, Pan L, Du J, et al. Prevalence of, and biochemical and anthropometric risk factors for, dyslipidemia in children and adolescents aged 7 to 18 years in China: A cross-sectional study. Am J Hum Biol 2019;31:e23286.

19. He H, Pan L, Du J, et al. Muscle fitness and its association with body mass index in children and adolescents aged 7-18 years in China: a cross-sectional study. BMC Pediatr 2019;19:101.

20. Tanita BC420MA body composition analyser. Avaliable online: http://tanita.eu/media/wysiwyg/manuals/medicalapproved-body-compositionmonitors/bc-420mainstruction-manual

21. dos Santos Cavalcanti CB, do Egito Carvalho SCB, de Barros MVG. Anthropometric indicators of abdominal obesity: review of the papers indexed on SciELO electronic library. Braz J Kinathrop Hum Perform 2009. doi: 10.1590/1980-0037.2009v11n $2 \mathrm{p} 217$.

22. Sillanpää E, Cheng S, Häkkinen K, et al. Body composition in 18- to 88-year-old adults--comparison of multifrequency bioimpedance and dual-energy X-ray absorptiometry. Obesity 2014;22:101-9.

23. Chula de Castro JA, Lima TR, Silva DAS. Body composition estimation in children and adolescents by bioelectrical impedance analysis: A systematic review. J Bodyw Mov Ther 2018;22:134-46.

24. Franssen FM, Rutten EP, Groenen MT, et al. New reference values for body composition by bioelectrical impedance analysis in the general population: results from the UK Biobank. . J Am Med Dir Assoc 2014;15:448.e1-6.

25. Lu Y, Shu H, Zheng Y, et al. Comparison of fat-free mass index and fat mass index in Chinese adults. Eur J Clin Nutr 2012;66:1004-7.

26. Dallaire F, Sarkola T. Growth of Cardiovascular Structures from the Fetus to the Young Adult. Adv Exp Med Biol 2018;1065:347-60.

27. Kwok MK, Leung GM, Schooling CM. Pubertal testis volume, age at pubertal onset, and adolescent blood pressure: Evidence from Hong Kong's "Children of 1997" birth cohort. Am J Hum Biol 2017. doi: 10.1002/ ajhb.22993.

28. Kuciene R, Dulskiene V. Associations between body mass index, waist circumference, waist- to-height ratio, and high blood pressure among adolescents: a cross-sectional study. Sci Rep 2019;9:9493.

29. Wang M, Kelishadi R, Khadilkar A, et al. Body mass index percentiles and elevated blood pressure among children and adolescents. J Hum Hypertens 2020;34:319-25.

30. Yang Y, Dong B, Wang S, et al. Prevalence of high blood pressure subtypes and its associations with $\mathrm{BMI}$ in Chinese children: a national cross-sectional survey. BMC Public Health 2017;17:598.

31. Zhang YX, Wang ZX, Zhao JS, et al. Profiles of blood pressure among children and adolescents categorized by BMI and waist circumference. Blood Press Monit 2016;21:295-300.

32. Freedman DS, Goodman A, Contreras OA, et al. Secular Trends in BMI and Blood Pressure Among Children and Adolescents: The Bogalusa Heart Study. Pediatrics 2012;130:e159-66.

33. LA de Hoog M, van Eijsden M, Stronks K, et al. Association between body size and blood pressure in children from different ethnic origins. Cardiovasc Diabetol 2012;11:136.

34. Zhang YX, Wang SR. Distribution of body composition 
index and the relationship with blood pressure among children aged 7 to 12 years in Shandong, China. Asia Pac J Public Health 2012;24:981-8.

35. Yao XJ, Chen Z, Zhang GY. A study on body fat in children aged 7-12. Zhonghua Yu Fang Yi Xue Za Zhi

Cite this article as: $\mathrm{He} \mathrm{H}, \mathrm{Pan} \mathrm{L}$, Du J, Jin Y, Wang L, Jia P, Shan G. Effect of fat mass index, fat free mass index and body mass index on childhood blood pressure: a cross-sectional study in south China. Transl Pediatr 2021;10(3):541-551. doi: $10.21037 /$ tp-20-325
1994;28:213-5.

36. Flynn JT, Kaelber DC, Baker-Smith CM, et al. Clinical Practice Guideline for Screening and Management of High Blood Pressure in Children and Adolescents. Pediatrics 2017;140:e20171904. 